\title{
Suitability of 3D-Printed devices for low-temperature geochemical experiments
}

\author{
Karel Kletetschka ${ }^{\mathrm{a}}$, J. Donald Rimstidt ${ }^{\mathrm{a}}$, Timothy E. Long ${ }^{\mathrm{b}, \mathrm{c}}$, F. Marc Michel ${ }^{\mathrm{a}, \mathrm{b}, \mathrm{d}, *}$ \\ a Department of Geosciences, Virginia Polytechnic Institute and State University, Blacksburg, VA, 24061, USA \\ ${ }^{\mathrm{b}}$ Virginia Polytechnic Institute and State University, Macromolecules Innovation Institute (MII), USA \\ ${ }^{\mathrm{c}}$ Department of Chemistry, Virginia Polytechnic Institute and State University, Blacksburg, VA, 24061, USA \\ ${ }^{\mathrm{d}}$ Virginia Tech Academy of Integrated Science, Division of Nanoscience, USA
}

\section{A R T I C L E I N F O}

Editorial handling by Prof. M. Kersten

Keywords:

3D printing

Solvent resistance

Aqueous geochemistry

Polymer stability

Stereolithography

\begin{abstract}
A B S T R A C T
Desktop 3D printing stereolithography (SLA) is a fabrication technique based on photopolymerization that can be used to efficiently create novel reaction devices for laboratory geochemistry with complex features (e.g. internal channels, small volumes) that are beyond the capabilities of traditional machining methods. However, the stability of 3D printed parts for low-temperature aqueous geochemical conditions has not been carefully evaluated. Furthermore, it is unclear what criteria should be used when attempting to optimize the mechanical and chemical properties during post-processing steps. Addressing these challenges is important for determining the suitability of 3D printed devices for laboratory investigations such as mineral precipitation/dissolution experiments. Here, we use thermogravimetric analysis (TGA) profiles, dynamic mechanical analysis (DMA), and chemical extraction of leachables to show how ultraviolet (UV) post-curing can optimize properties of a commercial photo-reactive resin (Formlabs Standard Clear). The mechanical and chemical stability of the post-cured material was enhanced and a working temperature of up to $80^{\circ} \mathrm{C}$ was determined. We further provide data showing the stability and compatibility of the material in aqueous conditions of $\mathrm{pH} 0,5.7$ and 12. As SLA 3D printing is still an emerging and rapidly developing technology, the method presented here will provide a framework for assessing how new printer types and materials (i.e. resins) impact the suitability of SLA printed devices for future experimental studies.
\end{abstract}

\section{Introduction}

We have recently showed how desktop 3D printing stereolithography (SLA) can be used to manufacture small volume chemical reactors for laboratory geochemical kinetics and synthesis experiments (Michel et al., 2018). These devices are inexpensive, rapidly produced, and highly customizable relative to conventional machining methods. For experimental geochemists in particular, the ability to fabricate complex geometries, flow paths, and mixing conditions coupled with real-time analytical techniques is extremely valuable. However, the suitability of SLA 3D printed materials for use at relevant aqueous geochemical conditions is not established. In particular, it is imperative that we know its chemical, mechanical, and thermal stability to ensure that the 3D printed material does not degrade during use or influence experimental results. The overall objective of this work is to develop a way to optimize and test solvent resistance and mechanical stability of SLA 3D printed polymers. This study focused on the behavior of one type of SLA 3D printed polymer at experimental conditions relevant to low temperature aqueous geochemistry. However, this information will also be useful for other fields of research already using devices fabricated by 3D printing in experimental studies. For example, lithographic 3D printed microfluidics devices are now being used in chemical engineering, biomedical and organic chemistry research. (Au et al., 2016; Dragone et al., 2013; Okafor et al., 2017).

Stereolithography is a laser-based additive manufacturing process that uses an ultraviolet (UV)-sensitive material to convert 3D digital models into functional prototypes. Current desktop SLA 3D printers use methacrylate-based photopolymer resins that consist of varying ratios of monomers, oligomers, and a photoinitiator. Ultraviolet light from the laser is absorbed by the photoinitiator, generating a radical which initiates polymerization of the resin, initiating a chain reaction that propagates until termination, forming the polymer chain (Mishra and Yagci, 2016). Crosslinking of the polymer chains also happens during the reaction, leading to rapid solidification of the liquid resin. The

\footnotetext{
* Corresponding author. Department of Geosciences, Virginia Polytechnic Institute and State University, Blacksburg, VA, 24061, USA.

E-mail address: mfrede2@vt.edu (F.M. Michel).
} 
overall sequence of reactions allows solid 3D models to be built rapidly layer-by-layer. By design, SLA printers produce polymers that do not fully convert and cross-link by the UV laser so a post-curing procedure that exposes the printed parts to additional UV light is used to further the crosslinking process towards completion. Post-curing is known to improve mechanical properties of SLA printed polymers and similar photopolymers (Watters and Bernhardt, 2017).

Methacrylate-based photopolymers are generally considered to be mechanically and chemically stable in non-organic solvents. Much of the current literature about methacrylate photopolymer behaviors in aqueous media comes from researchers interested in developing dental composites (Ferracane, 1994; Inoue and Hayashi, 1982; Kloukos et al., 2013; Tanaka et al., 1991). SLA materials differ from dental composites in that they require specific viscosities, functionalities, and cure times to be suitable for lithographic printing (Mondschein et al., 2017; Sirrine et al., 2018; Tahayeri et al., 2016). Parameters such as degree of crosslinking have been also shown to have a significant effect on water sorption behavior and material leachability in methacrylate photopolymers used in other applications (Ferracane, 1994; Krongauz, 2010; Parthasarathy et al., 2012; Rueggeberg and Craig, 1988).

Mechanical stability and solvent resistance are essential if SLA 3D printed materials are to be used in geochemical experiments. Prior work showed that mechanical properties of SLA materials improved after post-curing, including for the Formlabs Standard Clear resin (Bennett, 2017; Zguris, 2018). Those studies were unconcerned with applications in laboratory equipment that is exposed to chemical solutions, so they lack information beyond basic mechanical strength. Moreover, previous studies are inconsistent in methodology and often require the use of complex instrumentation that can be cost prohibitive. There has been some work utilizing the Formlabs Standard Clear polymer resin for use in microfluidic devices in which the researchers conduct short term exposures of the material to organic solvents followed by thermomechanical stability testing. These studies found little evidence of incompatibility or degradation (Okafor et al., 2017). However, they were short-term $(\leq 24 \mathrm{~h})$ exposures and issues associated with unreacted species within the polymer were not addressed.

In this study, we used thermal, chemical and mechanical tests to evaluate the mechanical stability and solvent resistance of an SLA printed material (Formlabs Standard Clear) before and after UV postcuring, as well as after aging the material in aqueous solutions for extended periods. Thermogravimetric analysis (TGA), dynamic mechanical analysis (DMA), and chemical extraction to estimate leachable fraction were identified as useful methods for evaluating changes in mechanical stability and solvent resistance. TGA and DMA measurements were used to assess the effect of UV post-curing time. Since postcuring should increase the conversion and crosslinking of the photopolymer, the insoluble crosslinked fraction of the material (gel fraction) should also increase. The gel fraction was determined by extracting the soluble (sol) fraction with chloroform, a strong organic solvent. Thermomechanical and chemical extraction results were used to optimize the post-curing procedure for this particular printer and resin as well as to determine a working temperature for the material. Post-cured test pieces were then exposed to aqueous solutions ranging from strongly acidic to strongly basic $\mathrm{pH}$ for extended periods to test mechanical stability and solvent resistance. Overall, the results significantly improve our understanding of the behaviors of SLA 3D printed polymers by addressing key questions regarding their suitability for use at low temperature aqueous conditions.

\section{Materials and methods}

\subsection{Test piece design and fabrication}

Three types of test parts were used for polymer testing. The parts for dynamic mechanical analysis (DMA) were rectangular bars with dimensions of $25 \times 5 \times 1 \mathrm{~mm}$ (length, width, thickness). The parts for thermogravimetric analysis (TGA) were printed as $1 \mathrm{~mm}$ thick disks with a $4 \mathrm{~mm}$ diameter. Test parts for gel fraction and leachability tests were square plates with dimensions of $25 \times 25 \times 1 \mathrm{~mm}$. All parts were designed using the 3D modeling software form $\mathrm{Z}$ Pro (AutoDesSys, Inc.) and exported as stereolithography (.stl) files. Images and downloadable stl files for the test parts are available in Supporting Information.

All parts were printed with a Formlabs Form 2 printer using the Standard Clear (V2-FLGPCL02) resin with a layer thickness of $50 \mu \mathrm{m}$. The stl files were prepared for printing using the printer software PreForm (Formlabs, v. 2.14.0). This step included model repair (if needed) and slicing, as well as orienting the models and adding supports. All parts were printed with the long axis of the part perpendicular to the build platform and supports were added automatically using PreForm. Detailed instructions for these steps are described elsewhere (Michel et al., 2018). After printing, all parts were placed in a bath of isopropanol (IPA, 99\%, VWR) for 12 min with agitation by hand for the first $2 \mathrm{~min}$. The parts were then transferred to a second fresh IPA bath for another $10 \mathrm{~min}$. Once removed, compressed air was used to remove excess IPA and the parts were then allowed to dry in air at room temperature. Unless otherwise stated, all parts were post-cured for a period of $3 \mathrm{~h}$ at $\sim 35^{\circ} \mathrm{C}$ in a custom built post-cure chamber containing 275 multi-directional 395-405 nm LEDs (Michel et al., 2018).

For comparison, another set of test pieces of the same dimensions was also fabricated using traditional machine milling of cast acrylic for chemical stability testing (see Supporting Information).

\subsection{Post-curing tests}

Parts were post-cured in UV light for periods ranging from 0 to $6 \mathrm{~h}$ to evaluate the effects of post-cure time on thermal and mechanical properties. Parts that were not post-cured were stored in the dark immediately after soaking in IPA and drying in air. Parts that were postcured for 1,3 , or $6 \mathrm{~h}$ were also stored in the dark prior to analysis. The results of post-curing tests were used to determine the optimal amount of post-curing time for the parts used in subsequent $\mathrm{pH}$ stability tests.

\subsection{Chemical stability tests}

Parts washed in IPA and post-cured for $3 \mathrm{~h}$ were aged individually in acidic, neutral, or basic aqueous solutions for different periods of times to test the stability of the 3D printed polymers. Test parts were completely immersed in $5 \mathrm{~mL}$ of either an acidic solution $\left(1 \mathrm{M} \mathrm{HNO}_{3}, \mathrm{pH}\right.$ 0.07), near neutral (DI water, $\mathrm{pH} 5.70$ ), and basic $(0.01 \mathrm{M} \mathrm{NaOH}, \mathrm{pH}$ 12.06) for 1 ( $24 \mathrm{~h}), 7(168 \mathrm{~h})$, or 18 (432 h) days. Sodium hydroxide solutions were made by dissolving $80 \mathrm{mg} \mathrm{NaOH}$ in $200 \mathrm{~mL}$ of deionized water. Nitric acid solutions were made by diluting $12.7 \mathrm{~mL}$ of concentrated $\mathrm{HNO}_{3}(70 \%)$ to $200 \mathrm{~mL}$ with deionized water. All chemicals used were reagent grade (Sigma-Aldrich). Samples removed at the desired aging time were dabbed dry using KimWipes, weighed, dried in a vacuum oven $\left(40^{\circ} \mathrm{C}, 40 \mathrm{~h}\right)$ and then reweighed. The tests were done in triplicate. The parts were stored in the dark prior to thermal and mechanical analysis. The chemical stability tests were also performed on machined cast acrylic test pieces for comparison (Supplementary Information).

\subsection{Polymer analysis}

\subsubsection{Thermal analysis}

Thermogravimetric Analysis (TGA) was performed using a TA Instruments (Newcastle, DE) Q500 $\left(10^{\circ} \mathrm{C} \mathrm{min}^{-1}, \sim 20-600^{\circ} \mathrm{C}\right)$. TGA was performed in air for the parts used to evaluate the effects of postcuring time. The presence of air promotes char formation, which is related of degree of crosslinking in methacrylate-based polymers (Lomakin et al., 1993). TGA for $\mathrm{pH}$ susceptibility experiments was done under nitrogen $\left(\mathrm{N}_{2}\right)$ gas to limit oxidative effects not associated with solvent exposure. 


\subsubsection{Dynamic mechanical analysis}

Dynamic mechanical analysis (DMA) was done using a TA Instruments Q800 Multifrequency (Strain) Analyzer at a frequency of $1 \mathrm{~Hz}$ in air. An amplitude of $15 \mu \mathrm{m}$ and pre-load of $0.01 \mathrm{~N}$ were used in a temperature sweep from $30^{\circ}-220^{\circ} \mathrm{C}$ at $3{ }^{\circ} \mathrm{C} \mathrm{min}{ }^{-1}$. The location of the peak of the $\tan \delta$ vs. temperature curve was used as the glass transition temperature $\left(\mathrm{T}_{\mathrm{g}}\right)$ and the storage modulus at $150{ }^{\circ} \mathrm{C}$ was used as the rubbery modulus $\left(E^{\prime}\right)$ - the storage modulus value at which it plateaus. The crosslink density $\left(\mathrm{V}_{\mathrm{e}}\right)$ was calculated using those parameters in Flory's rubbery elasticity theory equation (Krongauz, 2010):

$V_{e}=\frac{E^{\prime}}{3 R T_{g}}$

where $\mathrm{R}$ is the universal gas constant $\left(8.314 \mathrm{~J} \mathrm{~mol}^{-1} \mathrm{~K}^{-1}\right)$. The rubbery modulus for our samples is in the appropriate range of $2 \times 10^{6} \mathrm{~Pa}$ to $2 \times 10^{8} \mathrm{~Pa}$ for this equation to be applicable (Krongauz, 2010; Parthasarathy et al., 2012).

\subsubsection{Gel fraction \& leachables}

Determination of the soluble fraction of polymer was done by a chloroform extraction. A set of uncured printed test plates and a set of 3-h post-cured plates were submerged in $150 \mathrm{~mL}$ of stirred chloroform ( $0.75 \%$ ethanol, Fisher Scientific) for $40 \mathrm{~h}$. Chloroform was replaced at 2,5 , and $24 \mathrm{~h}$ from start time. The samples were then removed and dried in a vacuum oven $\left(70{ }^{\circ} \mathrm{C}, 40 \mathrm{~h}\right)$. Dry masses before and after were used to calculate gel fraction (final mass/initial mass).

\section{Results}

\subsection{Effects of post-curing on mechanical properties}

\subsubsection{Thermal analysis}

Weight loss profiles as a function of temperature from TGA of uncured and post-cured polymer test pieces are shown in Fig. 1a. Two main weight loss regimes are observed. The first regime, which starts at $\sim 220^{\circ} \mathrm{C}$, has been attributed to partial loss of the side groups of the methacrylate polymer chain and the second regime, at $\sim 340^{\circ} \mathrm{C}$, has been attributed to depolymerization (Okafor et al., 2017).

The onset of degradation occurs at a lower temperature in the uncured parts than in each of the post-cured parts. The average mass remaining (char) of the polymer at $495{ }^{\circ} \mathrm{C}$ (Fig. $1 \mathrm{~b}$ ) increases from $0.0 \%$ to $6.5 \%$ after 1 -h post-cure time, $6.6 \%$ after $3 \mathrm{~h}$ of post-curing, and $5.0 \%$ after $6 \mathrm{~h}$. Based on our replicates, these measurements show an associated error of up to $\sim \pm 1.5 \%$.

\subsubsection{Dynamic mechanical analysis}

DMA data from the temperature sweeps of the uncured part

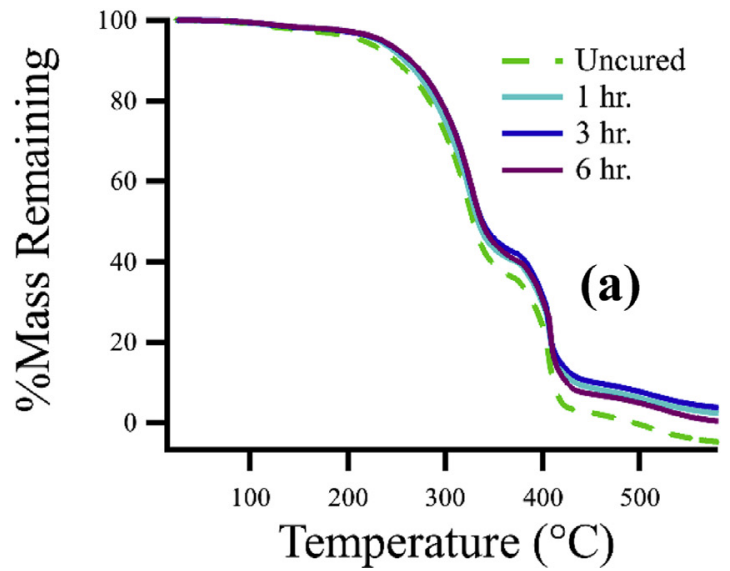

compared to parts post-cured for $3 \mathrm{~h}$ are shown in Fig. 2. The profiles show a storage modulus plateau at $\sim 12.2 \mathrm{MPa}$ and $\sim 17.2 \mathrm{MPa}$ for the uncured and 3-h post-cured samples, respectively (Fig. 2a). The glass transition occurs at $\sim 94^{\circ} \mathrm{C}$ and $\sim 108^{\circ} \mathrm{C}$ for the uncured and postcured samples, respectively (Fig. 2b).

Crosslink density values $\left(\mathrm{V}_{\mathrm{e}}\right)$ calculated from the thermal and mechanical parameters obtained from DMA are listed in Table 1.

\subsection{Chemical stability}

Exposure of printed test pieces to nitric acid, DI water, or sodium hydroxide for a period of 18 days resulted in no visible swelling or deformation in any of the samples. Thermal analysis of the samples exposed to the three solutions (Fig. 3) shows overlapping degradation curves for all samples including the control. Minimal differences were observed for all tested conditions. Thermal analysis of machined cast acrylic test pieces is shown for comparison (Supplementary Information, Figure S1).

Results of the DMA temperature sweep of samples exposed to the three solutions for 18 days are shown in Fig. 4. The $\tan \delta$ vs. temperature curves show the glass transitions occurring at $\sim 108^{\circ} \mathrm{C}$, $\sim 113^{\circ} \mathrm{C}, \sim 111^{\circ} \mathrm{C}$, and $\sim 111^{\circ} \mathrm{C}$ for the control, $\mathrm{pH} \mathrm{0,pH} 6$, and $\mathrm{pH} 12$ samples, respectively. Corresponding storage moduli (Table 2) plateau at $\sim 17.2 \mathrm{MPa}, \sim 20.2 \mathrm{MPa}, \sim 19.0 \mathrm{MPa}$, and $\sim 16.5 \mathrm{MPa}$.

DMA of machined cast acrylic shown for comparison (Supplementary Information, Figure S2) shows a higher $\mathrm{T}_{\mathrm{g}}$ of $\sim 148^{\circ} \mathrm{C}$ and no changes after exposure to the different solutions for 18 days.

\subsection{Gel fraction \& leachables}

The chloroform exposure resulted in significant observable swelling and fracture of the parts. Results for the fraction of the non-leachable material in the uncured and 3-h post-cured parts are shown in Table 3. The non-leachable fraction is assumed to be part of the cross-linked insoluble network.

\section{Discussion}

\subsection{Post-curing}

In order to identify an optimum post-curing procedure for enhancing resistance of 3D printed reactors made of Standard Clear resin (Formlabs) to aqueous conditions and solvent infiltration, an experiment testing thermomechanical properties as a function of UV post-cure time was conducted. Thermogravimetric analysis of uncured test piece (Fig. 1) showed an onset of degradation at lower temperatures than the 1,3 , and 6-h post-cured samples. Previous thermogravimetric studies

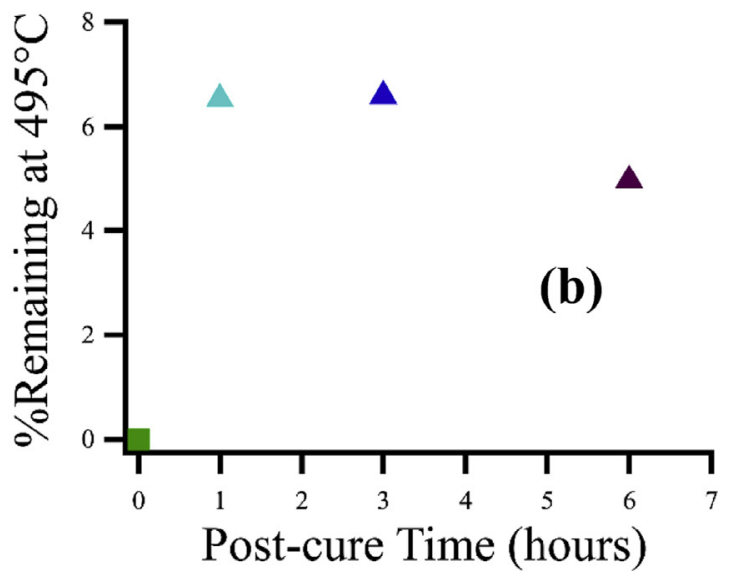

Fig. 1. (a) TGA weight loss profiles for parts post-cured for 0 (uncured), 1,3 , and $6 \mathrm{~h}$ (b) $\%$ mass remaining at $495{ }^{\circ} \mathrm{C}$ for each part. 

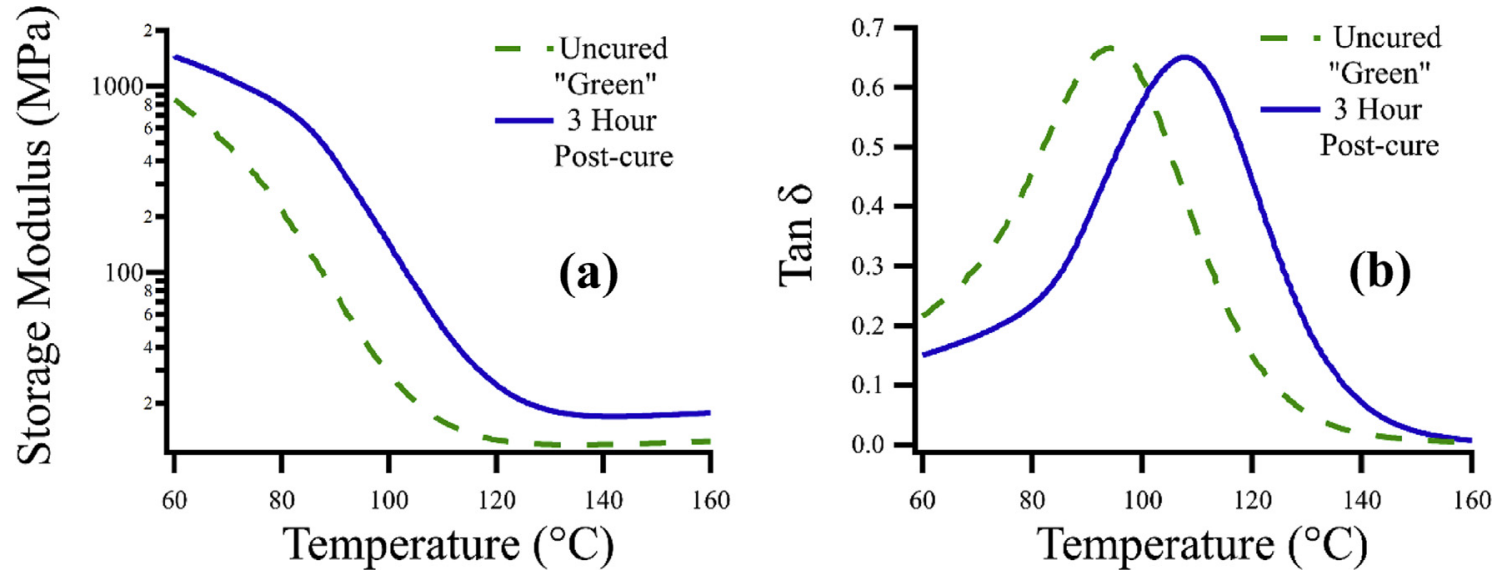

Fig. 2. DMA temperature sweep profiles showing (a) storage modulus vs. temperature and (b) tan $\delta$ vs. temperature of uncured and 3-h post-cured test parts.

Table 1

Glass transition temperatures $\left(\mathrm{T}_{\mathrm{g}}\right)$, rubbery moduli ( $\left.\mathrm{E}^{\prime}\right)$, and crosslink density values $\left(\mathrm{V}_{\mathrm{e}}\right)$ from DMA analysis of uncured and $3 \mathrm{~h}$ post-cured test pieces.

\begin{tabular}{llll}
\hline Sample & $\mathrm{T}_{\mathrm{g}}\left({ }^{\circ} \mathrm{C}\right)$ & $\mathrm{E}^{\prime}(\mathrm{Pa})$ & $\mathrm{V}_{\mathrm{e}}\left(\mathrm{mol} / \mathrm{m}^{3}\right)$ \\
\hline Uncured & 94 & $1.22 \times 10^{7}$ & 1330.54 \\
3-h Post-cure & 108 & $1.72 \times 10^{7}$ & 1806.87 \\
\hline
\end{tabular}

showed that poorly crosslinked methacrylate-based photopolymers degrade at lower temperatures when compared to their crosslinked counterparts (Rychlý and Pavlinec, 1990). Residual mass at $495^{\circ} \mathrm{C}$ in our samples was significantly higher at around $6 \%$ (compared to $0 \%$ in uncured control) in all of the post-cured samples, with the highest at $6.6 \%$ mass remaining in the 3-h post-cured sample. Residual mass, known as "char", is known to increase with crosslink density (Lomakin et al., 1993) indicating that our 3-h post-cure time achieved the highest crosslink density of the sample set, although the results suggest that even after $1 \mathrm{~h}$ the degree of crosslinking was nearly maximized.

The glass transition temperature $\left(\mathrm{T}_{\mathrm{g}}\right)$ of polymers corresponds with the temperature at which the polymer chains transition into a rubbery state, where they are increasingly mobile and can slide past each other. Increasing $\mathrm{T}_{\mathrm{g}}$ increases the temperature range over which the polymer retains its optimal mechanical properties, such as hardness and rigidity. DMA results (Fig. 2) show that post-curing increases the $\mathrm{T}_{g}$ by $14^{\circ} \mathrm{C}$. The beginning of the glass transition peak can be used to approximate the maximum safe operating temperature for the material and was found to be $\sim 80^{\circ} \mathrm{C}$ for the post-cured sample. The increase in $\mathrm{T}_{g}$ after post-curing has been shown in other methacrylate materials and attributed to an increase in crosslink density of the polymer (Alves et al., 2004). Our post-cured samples also show an increase in rubbery modulus of $\sim 5 \mathrm{MPa}$, also indicating higher mechanical stability. Applying both measured parameters in Flory's rubber elasticity equation for crosslink density, the 3-h post-cured sample showed a $35.8 \%$ increase in crosslink density (Table 1). This significant increase is important considering that resistance to solvent infiltration and swelling increases with crosslink density. Longer post-curing times did not result in a significant increase in cross-link density, which is why $3 \mathrm{~h}$ was chosen as the optimal post-cure time for $3 \mathrm{D}$ printed pieces used in $\mathrm{pH}$ stability tests.

\subsection{Chemical stability}

Mechanical properties tests were used to evaluate the stability of the 3D printed test pieces incubated in solutions with different $\mathrm{pH}$ for periods of up to 18 days. TGA profiles of the samples showed virtually no difference with incubation time or $\mathrm{pH}$ and were virtually identical to the control. Differences in the TGA curves would be expected if the mechanical properties had changed due to degradation (Pandey et al., 2005). DMA results (Fig. 4) also show no evidence of changes in the bulk mechanical properties due to material degradation. Rather, the results show that aging in water causes a slight increase in stability as indicated by increases in both $\mathrm{T}_{\mathrm{g}}$ and rubbery modulus. We attribute increases in these properties to minor leaching of compounds unattached to the primary polymer network such as unreacted monomers and oligomers. Regardless of crosslinking method, all polymers are only partially cross-linked and the unattached molecules are likely leached

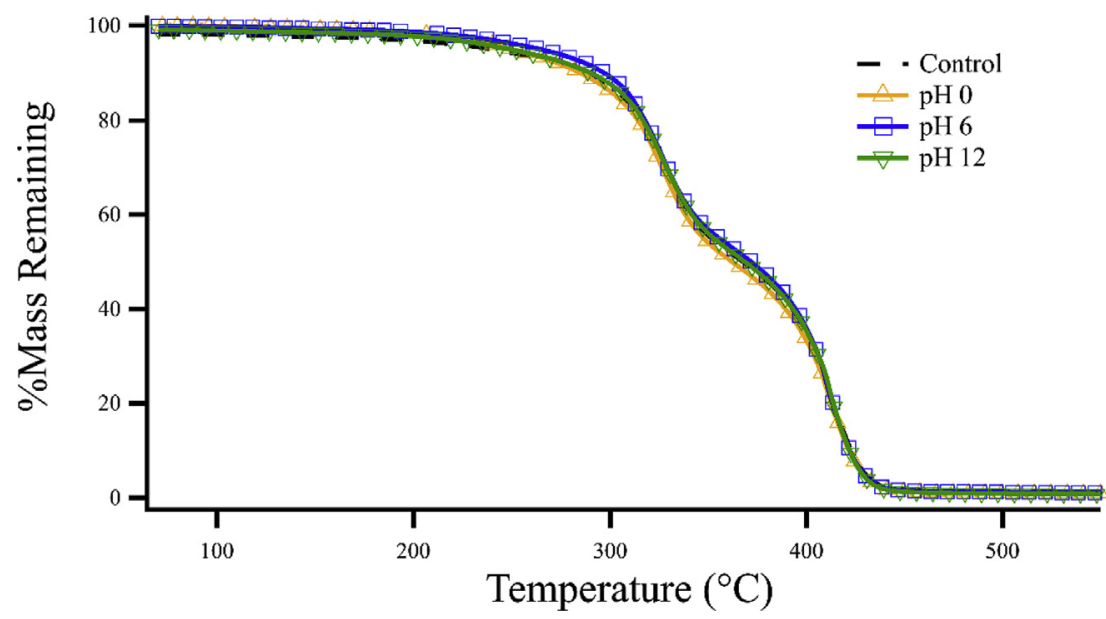

Fig. 3. TGA weight loss profiles of test parts after different $\mathrm{pH}$ exposures for 18 days. 

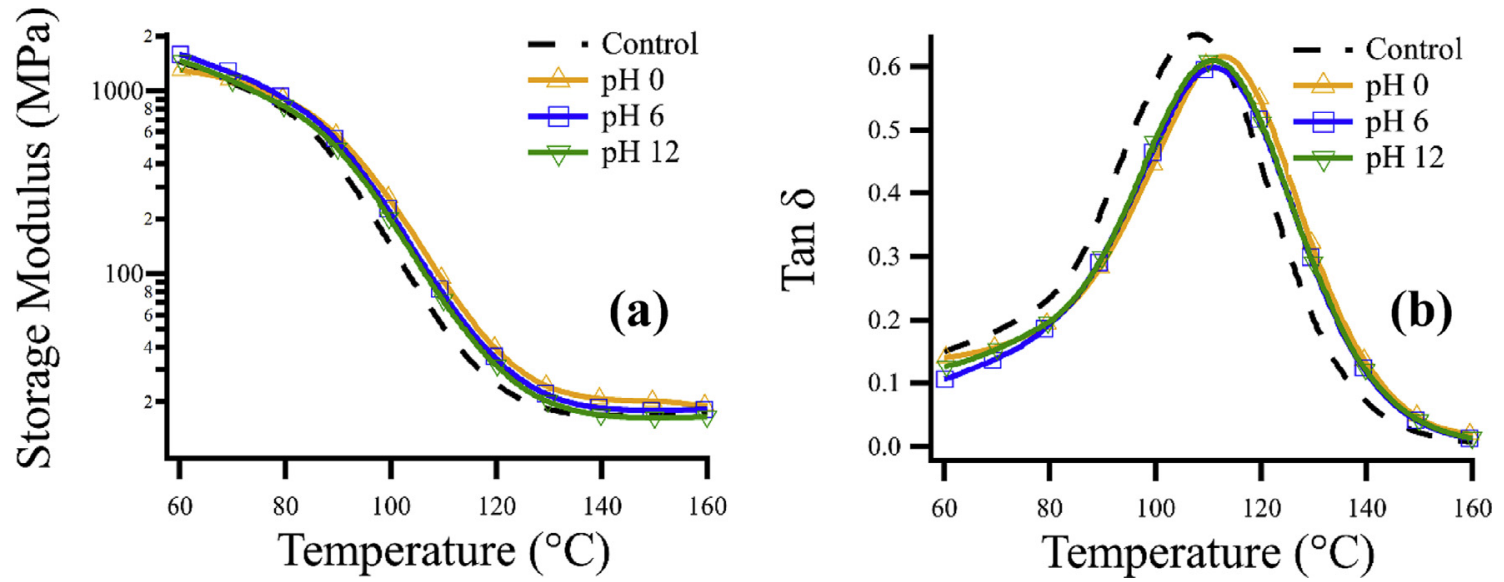

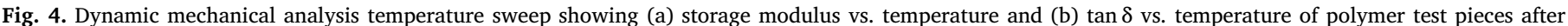
different $\mathrm{pH}$ exposures for 18 days.

Table 2

Glass transition temperature $\left(\mathrm{T}_{\mathrm{g}}\right)$ and rubbery modulus (E') for SLA printed test pieces after different $\mathrm{pH}$ exposures for 18 days.

\begin{tabular}{lcc}
\hline Sample & $\mathrm{T}_{\mathrm{g}}\left({ }^{\circ} \mathrm{C}\right)$ & $\mathrm{E}^{\prime}(\mathrm{Pa})$ \\
\hline Control & 108 & $1.72 \times 10^{7}$ \\
$\mathrm{pH} \mathrm{0}$ & 113 & $2.02 \times 10^{7}$ \\
$\mathrm{pH} \mathrm{7}$ & 111 & $1.90 \times 10^{7}$ \\
$\mathrm{pH} \mathrm{12}$ & 111 & $1.65 \times 10^{7}$ \\
\hline
\end{tabular}

Table 3

Gel fraction of the uncured and 3-h post-cured parts.

\begin{tabular}{ll}
\hline Sample & Gel Fraction \\
\hline Uncured & $0.902 \pm 0.003$ \\
3-h Post-cured & $0.967 \pm 0.001$ \\
\hline
\end{tabular}

out when exposed to a solvent for sufficient time (Nandi and Winter 2005). Leaching of unreacted monomers in aqueous environments has been discussed for a variety of methacrylate-based photopolymers used in dentistry (Ferracane, 1994; Inoue and Hayashi, 1982; Kloukos et al., 2013; Tanaka et al., 1991) and, more broadly, has been mentioned to be a possible environmental concern associated with plastic waste (Teuten et al., 2009). However, in SLA 3D printing this issue has been sparsely discussed but is of particular concern for applications involving experimental reactionware. In our measurements, the sample exposed to nitric acid shows a higher $\mathrm{T}_{\mathrm{g}}$ and $\mathrm{E}$ ' than the other samples, suggesting that the solution may be more effective at extracting the leachable material, however the difference is relatively minor and is close to or within instrumental error limits. The results show that the $3 \mathrm{D}$ printed reactor material can withstand aggressive acidic $(\mathrm{pH} 0)$ and basic $(\mathrm{pH}$ 12) conditions, which suggests that reactors can be cleaned and reused similar to acrylic and glass. Chemical stability experiments done for comparison on analogous machined cast acrylic test pieces show no apparent changes in the DMA curves (Supplementary Information, Figure S2). The $\mathrm{T}_{\mathrm{g}}$ for the machined acrylic parts was generally higher than our SLA parts at $\sim 148{ }^{\circ} \mathrm{C}$, indicating a higher working temperature, and remained unchanged after all 18 day exposures.

To identify the maximum extent of potential leaching in the 3D printed material, chloroform extraction measurements were used to determine the fraction that is part of the insoluble polymer network (gel) and the fraction that can potentially leach out (sol). The gel fractions of the uncured and 3-h post-cured samples were calculated as $90 \%$ and $97 \%$, respectively. This indicates that post-curing results in a polymer that is almost completely cross-linked. The remaining portion serves as an approximation of the maximum amount of material that could potentially leach out of the solid and into solution over an extended period of time.

\subsection{Recommendations for testing and use of $3 D$ printed reactors}

For reactions particularly sensitive to trace amounts of monomer methacrylate materials, it may be useful to allow the printed reactor(s) to leach out surface compounds in a bath of DI water or isopropanol. Prior studies of methacrylate photopolymers for dentistry (Ferracane, 1994) and preliminary tests of our SLA material suggest that water is not an effective solvent for "pre-leaching" of unreacted compounds but that isopropanol may be effective without excess swelling of the polymer. Stronger organic solvents such as chloroform are not recommended for treating reactors because of swelling behavior that can cause the material to become brittle and fracture. For most applications involving geochemistry, the concentrations and rates of removal of leachable monomers are so slow as to be unlikely to affect reactions, especially for experiments using mixed flow reactors (Blue et al., 2013; Rimstidt and Dove, 1986). It should be noted that since the $\mathrm{T}_{\mathrm{g}}$ in this polymer occurs around $108^{\circ} \mathrm{C}$, we recommend avoiding reaction temperatures above $80^{\circ} \mathrm{C}$. Considering that most low temperature geochemical experiments are carried out at $<60^{\circ} \mathrm{C}$, this restriction is not of particular concern for our application.

For researchers interested in higher temperature geochemistry, including biogeochemical experiments where higher temperatures are often required for sterilization, the material studied here would be unsuitable. Standard autoclave temperatures used for sterilization typically range from $120^{\circ} \mathrm{C}$ to $190{ }^{\circ} \mathrm{C}$ at which this resin would lose mechanical stability/integrity. Interestingly, there is a "High Temperature" resin available from Formlabs (FLHTAM01) that may be a suitable alternative for experimental devices requiring sterilization. However, we recommend stability and compatibility testing before use. It should also be noted that most proprietary resins for 3D printers have not been tested for biocompatibility and few have been certified (Bhattacharjee et al., 2016). The toxicity of leachates from various commercial resins including an older formulation of Standard Clear resin (Form 1) have been evaluated (Zhu et al., 2015) and results showed that water-soluble leachates from various materials were toxic to several vertebrate and invertebrate indicator organisms. This toxicity may possibly be minimized by decreasing the leachable fraction through post-curing and pre-leaching steps. Nevertheless, it is evident that additional research is needed to establish the suitability of 3D printed reactors for experiments involving living organisms.

Post-curing of material after printing will improve mechanical properties and thermal stability. The fraction of material that can leach into solution will also be significantly reduced by post-curing. As shown 
here, TGA, DMA, and extraction are several methods that can be used to test the effectiveness of post-curing and changes during aging. Of these, TGA can provide crude estimates of changes in thermal stability but is most error-prone. DMA is a very useful technique that can provide parameters such as $\mathrm{T}_{g}$ and $\mathrm{E}$ ' which increase upon post-curing. Moreover, these parameters can help guide researchers interested in conducting higher temperature experiments by providing a working temperature range. Finally, extraction with an aggressive organic solvent such as chloroform is a useful way to assess post-curing effectiveness without the need for complex instrumentation. This can be done simply by measuring the change in dry mass before and after exposure. In addition to post-curing, we recommend placing printed and post-cured devices in a stirred bath of excess solvent such as isopropanol or DI water (less effective) followed by drying in a vacuum oven. For optimal results, this should be done for a period of 1-2 days to allow residual monomers to pre-leach before the first use.

\section{Conclusions}

A method for testing and optimizing SLA 3D printed materials for use in specialty low-temperature geochemical reactors was presented. Several key techniques are highlighted for optimizing mechanical properties, solvent resistance, and for determining leachable content and maximum operating temperature. These techniques are demonstrated on a commercial SLA 3D printed material, Formlabs Standard Clear, and the results show enhanced material properties, a reduction in leachable content, and a maximum operating temperature of $\sim 80^{\circ} \mathrm{C}$ in UV post-cured material. We further show methods for assessing material compatibility in aqueous conditions of geochemical interest. The suitability of the Formlabs Standard Clear material for aqueous conditions of $\mathrm{pH} 0,6$, and 12 was demonstrated; recommendations for testing and optimizing future SLA materials for use in relevant geochemical conditions are put forth.

\section{Acknowledgments}

This material is based upon work supported by the National Science Foundation under Grant No. EAR-1451996 and CAREER-1652237, as well as the Virginia Tech National Center for Earth and Environmental Nanotechnology Infrastructure (NSF Cooperative Agreement 1542100). Several of the characterization techniques in this work would not have been possible without the help of Dr. Charles Carfagna and the Macromolecules Innovation Institute (MII) that is part of the Institute for Critical Technology and Applied Science (ICTAS) at Virginia Tech.

\section{Appendix A. Supplementary data}

Supplementary data related to this article can be found at https:// doi.org/10.1016/j.apgeochem.2018.08.012.

\section{References}

Alves, N., Ribelles, G., Tejedor, G., Mano, J., 2004. Viscoelastic behavior of poly (methyl methacrylate) networks with different cross-linking degrees. Macromolecules 37 (10), 3735-3744.

Au, A.K., Huynh, W., Horowitz, L.F., Folch, A., 2016. 3D-Printed microfluidics. Angew Chem. Int. Ed. Engl. 55 (12), 3862-3881.

Bennett, J., 2017. Measuring UV curing parameters of commercial photopolymers used in additive manufacturing. Additive Manufacturing 18, 203-212.

Bhattacharjee, N., Urrios, A., Kang, S., Folch, A., 2016. The upcoming 3D-printing revolution in microfluidics. Lab a Chip 16 (10), 1720-1742.

Blue, C.R., Rimstidt, J.D., Dove, P.M., 2013. A mixed flow reactor method to synthesize amorphous calcium carbonate under controlled chemical conditions. Meth. Enzymol. 532, 557-568 Elsevier.

Dragone, V., Sans, V., Rosnes, M.H., Kitson, P.J., Cronin, L., 2013. 3D-printed devices for continuous-flow organic chemistry. Beilstein J. Org. Chem. 9, 951-959.

Ferracane, J., 1994. Elution of leachable components from composites. J. Oral Rehabil. 21 (4), 441-452.

Inoue, K., Hayashi, I., 1982. Residual monomer (Bis-GMA) of composite resins. J. Oral Rehabil. 9 (6), 493-497.

Kloukos, D., Pandis, N., Eliades, T., 2013. Bisphenol-A and residual monomer leaching from orthodontic adhesive resins and polycarbonate brackets: a systematic review. Am. J. Orthod. Dentofacial Orthop. 143 (4), S104-S112 e102.

Krongauz, V.V., 2010. Diffusion in polymers dependence on crosslink density. J. Therm. Anal. Calorim. 102 (2), 435-445.

Lomakin, S., Brown, J.E., Breese, R.S., Nyden, M.R., 1993. An investigation of the thermal stability and char-forming tendency of cross-linked poly (methyl methacrylate) Polym. Degrad. Stabil. 41 (2), 229-243.

Michel, F.M., Rimstidt, J.D., Kletetschka, K., 2018. 3D printed mixed flow reactor for geochemical rate measurements. Appl. Geochem. 89, 86-91.

Mishra, M., Yagci, Y., 2016. Handbook of Vinyl Polymers: Radical Polymerization, Process, and Technology. CRC press.

Mondschein, R.J., Kanitkar, A., Williams, C.B., Verbridge, S.S., Long, T.E., 2017. Polymer structure-property requirements for stereolithographic 3D printing of soft tissue engineering scaffolds. Biomaterials 140, 170-188.

Nandi, S., Winter, H.H., 2005. Swelling behavior of partially cross-linked polymers: a ternary system. Macromolecules 38 (10), 4447-4455.

Okafor, O., Weilhard, A., Fernandes, J.A., Karjalainen, E., Goodridge, R., Sans, V., 2017. Advanced reactor engineering with 3D printing for the continuous-flow synthesis of silver nanoparticles. Reaction Chemistry \& Engineering 2 (2), 129-136.

Pandey, J.K., Reddy, K.R., Kumar, A.P., Singh, R., 2005. An overview on the degradability of polymer nanocomposites. Polym. Degrad. Stabil. 88 (2), 234-250.

Parthasarathy, R., Misra, A., Park, J., Ye, Q., Spencer, P., 2012. Diffusion coefficients of water and leachables in methacrylate-based crosslinked polymers using absorption experiments. J. Mater. Sci. Mater. Med. 23 (5), 1157-1172.

Rimstidt, J.D., Dove, P.M., 1986. Mineral/solution reaction rates in a mixed flow reactor. Wollastonite hydrolysis: Geochem. Cosmochim. Acta 50 (11), 2509-2516.

Rueggeberg, F., Craig, R., 1988. Correlation of parameters used to estimate monomer conversion in a light-cured composite. J. Dent. Res. 67 (6), 932-937.

Rychlý, J., Pavlinec, J., 1990. Thermal degradation of free radically prepared poly (methyl methacrylate). A nonisothermal weight loss study: Polym. Degrad. Stabil. 28 (1), $1-15$.

Sirrine, J.M., Meenakshisundaram, V., Moon, N.G., Scott, P.J., Mondschein, R.J., Weiseman, T.F., Williams, C.B., Long, T.E., 2018. Functional Siloxanes with Photoactivated, Simultaneous Chain Extension and Crosslinking for Lithography-based 3D Printing: Polymer.

Tahayeri, A., Fugolin, A., Ferracane, J., Pfeifer, C., Bertassoni, L., 2016. 3D printed versus conventionally cured experimental dental composites. Dent. Mater. 32, e90-e91.

Tanaka, K., Taira, M., Shintani, H., Wakasa, K., Yamaki, M., 1991. Residual monomers (TEGDMA and Bis-GMA) of a set visible-light-cured dental composite resin when immersed in water. J. Oral Rehabil. 18 (4), 353-362.

Teuten, E.L., Saquing, J.M., Knappe, D.R., Barlaz, M.A., Jonsson, S., Björn, A., Rowland, S.J., Thompson, R.C., Galloway, T.S., Yamashita, R., 2009. Transport and release of chemicals from plastics to the environment and to wildlife. Philos. Trans. R. Soc. Lond. B Biol. Sci. 364 (1526), 2027-2045.

Watters, M.P., Bernhardt, M.L., 2017. Curing parameters to improve the mechanical properties of stereolithographic printed specimens. Rapid Prototyp. J. 24 (1), 46-51.

Zguris, Z., 2018. How Mechanical Properties of Stereolithography 3D Prints Are Affected by UV Curing, vol. 4. Formlabs, Inc., Somerville, MA, pp. 2017 accessed Nov.

Zhu, F., Friedrich, T., Nugegoda, D., Kaslin, J., Wlodkowic, D., 2015. Assessment of the biocompatibility of three-dimensional-printed polymers using multispecies toxicity tests. Biomicrofluidics 9 (6), 061103. 